\title{
Aerobic fitness profile of youth soccer players: effects of chronological age and playing position
}

\section{Perfil aeróbio de jovens jogadores de futebol: efeitos da idade cronológica e da posição tática}

\author{
Giovani dos Santos Cunha \\ André Luís Lopes ${ }^{1}$ \\ Jeam Marcel Geremia ${ }^{1}$ \\ Gabriela Tomedi Leites ${ }^{2}$ \\ Bruno Manfredini Baroni ${ }^{3}$ \\ Rogério da Cunha Voser ${ }^{1}$ \\ Marco Aurélio Vaz ${ }^{1}$ \\ Álvaro Reischak-Oliveira ${ }^{1}$
}

Abstract - To establish a profile of the aerobic fitness in young soccer players, it is critical to consider different intervenient factors such as maturity status, chronological age and playing position. The aim of this study was to identify the biological maturation, chronological age, and playing position effects on physical and physiological characteristics of young soccer players. Two hundred and one soccer players of 11-19 years old were divided into groups relative to their maturity status, chronological age and playing position. A maximal exercise test was performed to determine peak oxygen uptake $\left(\mathrm{VO}_{2 \text { peak }}\right)$ and ventilatory thresholds $\left(\mathrm{VT}_{1}\right.$ and $\left.\mathrm{VT}_{2}\right)$ parameters in a treadmill. Biological maturation showed no significant effect on relative values $\left(\mathrm{mLkg}^{-1} \mathrm{~min}^{-1}\right)$ of $\mathrm{VO}_{2 \text { peak }}, \mathrm{VT}_{2}$ and $\mathrm{VT}_{1}\left(0.004<\eta^{2}\right.$ $<0.039$ ), but showed large positive effect on maximal aerobic speed (MAS) and speed at $\mathrm{VT}_{2}\left(\mathrm{VT}_{2 \text { speed }}\right)$. Chronological age showed a medium positive effect on relative values of $\mathrm{VO}_{2 \text { peak }}, V_{2} T_{2}$ and $\mathrm{VT}_{1}\left(0.095<\eta^{2}<0.137\right)$ and a large positive effect on MAS and $\mathrm{VT}_{2 \text { speed }}$. Relative values of $\mathrm{VO}_{2 \text { peak }}$ and $\mathrm{VT}_{1}$ showed no significant differences among groups for playing position $\left(\mathrm{P}>0.05 ; 0.044<\eta^{2}<0.051\right)$. However, goalkeepers showed significant lower relative values for $\mathrm{VT}_{2}$ and $\mathrm{VT}_{2 \text { speed }}$ than other playing positions and a medium positive effect was observed $\left(\mathrm{P}<0.05 ; 0.077<\eta^{2}<0.119\right)$. Chronological age showed a medium to large positive effect on aerobic fitness parameters, while biological maturation showed a positive effect only on MAS and $\mathrm{VT}_{2 \text { speed }}$. Playing position showed a medium positive effect on $\mathrm{VT}_{2}$ and $\mathrm{VT}_{2 \text { speed }}$.

Key words: Child; Puberty; Oxygen consumption; Anaerobic threshold; Running.

Resumo - Para identificar o perfil aeróbico de jovens jogadores de futebol é necessário considerar fatores intervenientes como maturação biológica, idade cronológica e posição tática. O objetivo do presente estudo foi identificar o efeito da maturação biológica, idade cronológica e posição tática sobre as caracteristicas físicas e fisiológicas de jovens jogadores de futebol. Duzentos e um jogadores de futebol com idades entre 11-19 anos foram divididos em grupos de acordo com estágio maturacional, idade cronológica e posição tática. Um teste máximo foi realizado para determinar o consumo de oxigênio de pico $\left(V O_{2 p i c o}\right.$ ) e limiares ventilatórios $\left(L V_{1}\right.$ e $\left.L V_{2}\right)$. A maturação biológica não apresentou efeitos significativos sobre os valores relativos $\left(\mathrm{mLikg}{ }^{-1} \cdot \mathrm{min}^{-1}\right)$ de $\mathrm{VO}_{2 \text { pico }}, L V_{2}$ e $L V_{1}\left(0,004<\eta^{2}<0,039\right)$, mas apresentou elevado efeito sobre velocidade aeróbia máxima (VAM) e velocidade em $L V_{2}\left(L V_{2 v e l o c i d a d}\right)$. A idade cronológica apresentou efeito positivo médio sobre os valores relativos de $V O_{2 \text { pico, }}^{\text {2velocidad }} V_{2}$ e $L V_{1}\left(0,095<\eta^{2}<0,137\right)$, e elevado efeito positivo sobre VAM e $L V_{\text {2velocidade }}$ A posiçấo tática não apresentou diferenças significativas sobre os valores relativos de $V \mathrm{O}_{2 \text { pico }}^{\text {2velociade }} \mathrm{L} V_{1}$ entre os grupos $\left(P>0,05 ; 0,044<\eta^{2}<0,051\right)$. Entretanto, goleiros apresentaram significativamente menores valores relativos de $L V_{2}$ e $L V_{\text {2velocidade }}$ em comparação com outras posiçôes táticas, com efeito positivo médio sendo identificado $\left(P<0,05 ; 0,077<\eta^{2}<\right.$ 0,119). A idade cronológica apresentou efeito positivo de médio a elevado sobre o perfil aeróbio, entretanto, a maturação biológica não apresentou efeito, exceto para VAM e $L V_{\text {2velocidade }}$. A posição tática apresentou efeitos positivos sobre $\mathrm{LV}_{2}$ e $\mathrm{LV}_{\text {2velocidade }}$

Palavras-chave: Criança; Puberdade; Consumo de oxigênio; Limiar anaeróbico; Corrida.
1 Universidade Federal do Rio Grande do Sul. Escola de Educação Física, Fisioterapia e Dança. Porto Alegre, RS. Brasil.

2 McMaster University. Child Health $\&$ Exercise Medicine Program. Hamilton, Ontario. Canadá.

3 Fundação Universidade Federal de Ciências da Saúde de Porto Alegre. Programa de Pós-Graduação em Ciências da Reabilitação. Porto Alegre, RS. Brasil.

Received: 05 June 2016 Accepted: 03 October 2016

\section{(C) (i) Licença}

Creative Commom 


\section{INTRODUCTION}

Soccer is one of the most popular sports in the world and it is characterized by a combination of physical, technical and tactical factors ${ }^{1,2}$. Soccer is an intermittent team sport involving low to high-intensity efforts ${ }^{3}$ and showing running as the main physical activity of the game ${ }^{1,3}$. During a match, the total running distance covered by a young soccer player ranges from 4 to $8.5 \mathrm{~km}^{1,3,4}$ while by a professional soccer player ranges from 8 to $12 \mathrm{~km}^{1}$. In terms of the physical performance during a soccer match, the aerobic fitness level has been considered as most powerful determinant of match running performance ${ }^{3}$. In addition, the distance run at high-intensity has been used to rank physical performance between players ${ }^{4,5}$.

In this context, the aerobic metabolism is important to soccer performance mainly because it is the predominant source of energy to supply physical demands ${ }^{1}$. For this reason, to determine aerobic fitness parameters such as peak oxygen uptake $\left(\mathrm{VO}_{2 \text { peak }}\right)$, ventilatory thresholds $\left(\mathrm{VT}_{1}\right.$ and $\left.\mathrm{VT}_{2}\right)$, maximal aerobic speed (MAS), speed at $\mathrm{VT}_{2}\left(\mathrm{VT}_{2 \text { speed }}\right)$ and $\mathrm{VT}_{1}$ $\left(\mathrm{VT}_{\text {1speed }}\right)$ are important to understand soccer performance and to identify intensity training zones and cardiorespiratory adaptations related to soccer training ${ }^{1-6} . \mathrm{VO}_{2 \text { peak }}$, MAS and $\mathrm{VT}_{2 \text { speed }}$ have showed a positive correlation with the total distance covered and high-intensity efforts performed by young soccer players during a match ${ }^{2,7}$. For example, physically fit players perform more high-intensity running during a match compared to theirs less-fit counterparts 7 . Improvements of $10 \%$ in $\mathrm{VO}_{2 \text { peak }}$ have been associated with a $20 \%$ increase in the total distance covered and twice the number of sprints during the game ${ }^{2}$. Additionally, $\mathrm{VT}_{2}$ have been considered an important variable to soccer performance mainly because it is very close to the intensity at which the soccer matches are played ${ }^{1,2} . \mathrm{VT}_{\text {2speed }}$ and $\mathrm{VT}_{\text {1speed }}$ have been used to delimit the high-intensity and low to moderate-intensity zones during match analysis, respectively ${ }^{5}$.

The interpretation of aerobic fitness features may be problematic from a talent identification/development perspective due to the biological maturational, chronological age, relative age and playing position effects. Some studies indicate that biological maturation can exert a positive effect on $\mathrm{VO}_{2 \text { peak }}{ }^{8-10}$ and ventilatory thresholds ${ }^{11}$. Consequently, biological maturation may be a confounding factor during the talent development and/or talent identification process. Athletes more advanced in biological maturation process were described with higher $\mathrm{VO}_{2 \text { eak }}$, power, speed, strength, sport-specific skills, and aerobic performance ${ }^{8,9}$. Consequently, late maturing boys are systematically excluded while early maturing boys are generally selected ${ }^{9,12,13}$.

Youth soccer competitions are generally organized into annual agegroups using specific cut-off values. This organization model was designed to give players equal participation and training opportunities, including fair opportunities for talent identification and/or development process ${ }^{13,14}$. However, soccer players from the same selection year can be older com- 
pared to their teammates. This can result in maturational and physical performance differences in favor of players born early in the birth year ${ }^{13,15,16}$. This phenomenon has been defined as relative age and its consequences are known as the relative age effect (RAE) ${ }^{13-15}$.

Players who are born early in the selection year (e.g. first birth quarter) take advantage of this subtle chronological lead and are more likely to be selected compared to their peers born later in the selection year (e.g. fourth birth quarter $)^{13}$. In this scenario, chronological age plays similar role as biological maturation favoring those athletes born in the first quarter and excluding those born in the fourth birth quarter. Therefore, these factors should be considered by soccer clubs to not bias the athlete's selection process towards the early maturing players due to the influences of $\mathrm{RAE}^{17,18}$.

In addition to biological maturation and chronological age, to establish an aerobic fitness profile in young soccer players, it is critical to consider other intervenient factors, such as playing position ${ }^{3}$. Playing position has been described as an important feature related to soccer performance in young soccer player due to its effects on aerobic fitness ${ }^{3,19-21}$. Currently literature lacks in studies about the effects of biological maturation, RAE and playing position on $\mathrm{VO}_{2 \text { peak }}$ and ventilatory thresholds parameters in high-level youth soccer players. So far, only few studies have investigated the relationship among aerobic fitness, biological maturation, RAE, and playing position in high-level young soccer players simultaneously. Therefore, the present study aimed to describe the physical and physiological profile of high-level young soccer players and to identify whether chronological age, maturity status, and playing position exert effects on $\mathrm{VO}_{2 \text { peak }}$ and ventilatory thresholds parameters.

\section{METHODOLOGICAL PROCEDURES}

\section{Participants}

This cross-sectional sample includes 201 high-level youth male soccer players aged 11-19 years old who were recruited from a professional club academy competing at the Brazilian national level. The participants were categorized into groups according to their maturity status, chronological age and playing position. All participants were engaged in formal training of 5 to 8 training sessions per week (60-120 minutes) and completed approximately one local game per week during the last competitive season. Inclusion criteria included at least 1 year of experience in formal soccer training. Participants were not taking any medications at the time of participation, reported no medical conditions including no history of surgery or musculoskeletal injury, and were not diagnosed with any chronic diseases. The study approval was obtained from the University Research Ethics Board (Project ID: 2008082) and was conducted in compliance with the standards set by the Declaration of Helsinki. Participants and their legal guardians were informed of the experimental protocol and potential risks and provided written informed assent and consent prior to participation. 


\section{Study Overview}

Participants visited the laboratory on a single session to complete several physical and physiological examinations, which included anthropometrics (height and body mass), determining maturity status, and completing an incremental exercise test to assess $\mathrm{VO}_{2 \text { peak }}$ and ventilatory thresholds parameters. They also completed a questionnaire about their health status, use of medication, birth date and years of training. Similar to previous studies ${ }^{20,22,23}$, all participants were separated into groups according to their self-reported best position in the field: goalkeeper (GK); full-back (FB); central-back (CB); defensive midfielder (DM); offensive midfielder (OM); and attacker (AT). Participants were familiar with the procedures before data collection commenced. All measurements were performed during the preseason period.

\section{Maturity Status}

Biological maturation was assessed by a trained healthcare professional using the criteria described by Tanner ${ }^{24}$. Prepubertal status was defined as stage I $(\mathrm{n}=26)$, pubertal as stage II $(\mathrm{n}=13)$, III $(\mathrm{n}=39)$, and IV ( $\mathrm{n}=$ $38)$, and as postpubertal as stage $V(n=85)$.

\section{Aerobic Fitness}

$\mathrm{VO}_{2 \text { peak }}$ and ventilatory thresholds parameters were assessed by open circuit spirometry (breath by breath) during the performance of a maximum treadmill running test. Following the manufacturer's instructions, the ergospirometry device (MedGraphics Cardiorespiratory Diagnostic Systems - CPX-D, St Paul, Minnesota) was manually calibrated using known gas concentrations. The participants were given time to adapt to the treadmill (Quinton Instruments-Seattle, USA) and ergospirometry device. Participants then performed a single progressive maximum effort test, which consisted of running for three minutes at $8 \mathrm{~km} \cdot \mathrm{hr}^{-1}$ followed by increases of $0.5 \mathrm{~km}^{-1} \mathrm{r}^{-1}$ every 30 seconds until exhaustion ${ }^{21}$. Participants were verbally encouraged during the test to achieve their maximal performance. To verify an exhaustive effort, each participant had to satisfy at least two of the following criteria upon termination of the treadmill test due to volitional exhaustion: 1) heart rate at the end of the test $\geq 95 \%$ of the predicted maximal heart rate, 2) a plateau of $\mathrm{VO}_{2}$ that was defined as an increase in $\mathrm{VO}_{2}$ of less than $2.1 \mathrm{~mL}^{-1} \mathrm{~kg}^{-1} \mathrm{~min}^{-1}$ with a corresponding increase in exercise intensity; 3) respiratory exchange ratio $(\mathrm{RER}) \geq 1.0^{21}$. Heart rate was measured using a heart rate monitor (Polar S610, USA). According to these criteria, all participants showed a valid $\mathrm{VO}_{2 \text { peak }}$.

$\mathrm{VO}_{\text {2peak }}$ was defined as the highest value recorded during the last minute of exercise test. When a plateau in $\mathrm{VO}_{2}$ could be detected, the higher value during plateau period was used to represent $\mathrm{VO}_{\text {2peak }}$. Ventilatory thresholds were determined in plots of the ventilation (VE), ventilatory equivalents $\left(\mathrm{VE} / \mathrm{VO}_{2}\right.$ and $\left.\mathrm{VE} / \mathrm{VCO}_{2}\right)$, partial pressure of end-tidal carbon dioxide $\left(\mathrm{PETCO}_{2}\right)$, and partial pressure of end-tidal oxygen $\left(\mathrm{PETO}_{2}\right)$ as a func- 
tion of oxygen uptake. Criteria to determine ventilatory thresholds were as follows: $\mathrm{VT}_{1}$ was determined when there was a rapid increase in the $\mathrm{VE} / \mathrm{VO}_{2}$ and $\mathrm{PETO}_{2}$ with no concomitant increase in $\mathrm{VE} / \mathrm{VCO}_{2}$ and $\mathrm{PETCO}_{2} . \mathrm{VT}_{2}$ was determined as the point at which a rapid rise in VE/ $\mathrm{VCO}_{2}$ and a fall in $\mathrm{PETCO}_{2}$ were observed. $\mathrm{VT}_{1}$ and $\mathrm{VT}_{2}$ were defined as the work rates associated with a first and a second nonlinear increase of $\mathrm{VE}$ and $\mathrm{VCO}_{2}{ }^{6,21}$. Three independent reviewers blindly determined ventilatory thresholds following the criteria previously described.

\section{Statistical analysis}

The normality of the distributions was investigated using the KolmogorovSmirnov test, and the homogeneity of the variables was assessed using Levene's test. The mean and standard deviation values were used in the descriptive analysis. Differences among maturity status, chronological age, and playing position groups for physical and physiological traits were tested with analysis of variance (One-way ANOVA). Bonferroni adjustments were done for post-hoc comparisons. For each ANOVA, partial eta-squared $\left(\eta^{2}\right)$ was calculated as a measure of effect size (ES). To interpret the magnitude of the effect size the following were adopted $<0.01$ as small, 0.06 to 0.15 as moderate and $>0.15$ as large effect ${ }^{25}$. Differences between the observed and expected birth-date distribution were tested with the Chi-square statistic. Expected birth-date distributions were estimated to be $25 \%$ for each quarter of the year. The level of statistical significance was set at $\mathrm{P}$ $\leq 0.05$. Statistical analyses were conducted using the statistical programs SPSS (version 19.0, SPSS, Inc., IBM Company; NY, USA).

\section{RESULTS}

Physical and physiological characteristics considering the entire group of high-level youth soccer players are presented in Table 1.

Table 2 depicts the physical and physiological characteristics in accordance to participant's biological maturation. As expected, postpubescent soccer players were significantly heavier and taller than prepubescent and pubescent soccer players $(\mathrm{P}<0.05)$. Biological maturation showed no significant effects on relative to body size values $\left(\mathrm{mL} \cdot \mathrm{kg}^{-1} \mathrm{~min}^{-1}\right)$ of $\mathrm{VO}_{2 \text { peak }}$, $\mathrm{VT}_{2}$ and $\mathrm{VT}_{1}\left(0.004<\eta^{2}<0.039\right)$; and no significant differences among maturational groups were observed $(\mathrm{P}>0.05)$.

Table 3 shows the physical and physiological characteristics in accordance to maturity status (Tanner stages). No significant differences were observed among maturational groups for relative to body mass values $(\mathrm{mL}$. $\left.\mathrm{kg}^{-1} \cdot \mathrm{min}^{-1}\right)$ of $\mathrm{VO}_{2 \text { peak }}, \mathrm{VT}_{2}$, and $\mathrm{VT}_{1}(\mathrm{P}>0.05)$. Exception was observed on the comparison between stages III and $\mathrm{V}$ for $\mathrm{VT}_{1}$ values $(\mathrm{P}<0.05)$. Tanner stages showed no significant effects relative to body mass values of $\mathrm{VO}_{2 \text { peak }}$ and $\mathrm{VT}_{2}\left(0.047<\eta^{2}<0.058\right)$. However, a medium positive effect was found for relative to body mass values of $\mathrm{VT}_{1}\left(\eta^{2}=0.067\right)$.

The groups comparisons based on their chronological age are presented 
in Table 4. Significant differences were found for relative to body mass values of $\mathrm{VO}_{2 \text { peak }}, \mathrm{VT}_{2}$ and $\mathrm{VT}_{1}$ manly in favor of older groups (13-14, 1516 , and > 17 years old) in comparison to the younger group (11-12 years old). Chronological age showed medium positive effect on relative to body mass values of $\mathrm{VO}_{2 \text { peak }}, \mathrm{VT}_{2}$ and $\mathrm{VT}_{1}\left(0.095<\eta^{2}<0.137\right)$.

Table 5 shows the physical and physiological characteristics according to playing position. Relative values $\left(\mathrm{mL} \mathrm{kg}^{-1} \cdot \mathrm{min}^{-1}\right)$ of $\mathrm{VO}_{2 \text { peak }}$ and $\mathrm{VT}_{1}$ showed no significant differences among groups for playing position $(\mathrm{P}>$ $\left.0.05 ; 0.044<\eta^{2}<0.051\right)$. However compared to the GK group, the FB, $\mathrm{DM}$ and $\mathrm{OM}$ groups showed significant differences for relative values of $\mathrm{VT}_{2}$, with a medium positive effect $\left(\mathrm{P}<0.05 ; \eta^{2}=0.077\right)$.

Interestingly, large positive effects of biological maturation status and chronological age on MAS and $\mathrm{VT}_{2 \text { speed }}$ were observed, while playing position showed a medium positive effect on $\mathrm{VT}_{\text {2speed }}$ (Tables 2, 3, 4 and 5).

$\mathrm{RAE}$ was identified due to overrepresentation of players born during the first semester of the year $(\mathrm{P}<0.05)$. Birth-date distribution by month quartile was $49.8 \%, 26.4,15.9 \%$ and $8 \%$ for $\mathrm{BQ}_{4}, \mathrm{BQ}_{2}, \mathrm{BQ}_{3}$, and $\mathrm{BQ}_{4}$, respectively.

Table 1. Physical and physiological characteristics of youth elite soccer players $(n=201)$

\begin{tabular}{|c|c|c|}
\hline Variable & Mean \pm SD & Minimum and maximal values \\
\hline Age (years) & $15.1 \pm 2.0$ & $11-19$ \\
\hline Body mass (kg) & $67.0 \pm 11.1$ & $37.9-94.3$ \\
\hline Height (cm) & $173.0 \pm 9.5$ & $148-195$ \\
\hline Years of training & $5.5 \pm 2.7$ & $1-12$ \\
\hline $\mathrm{VO}_{2 \text { peak }}\left(\mathrm{mL} \cdot \mathrm{min}^{-1}\right)$ & $4020 \pm 690$ & $2225-5510$ \\
\hline $\mathrm{VT}_{2}\left(\mathrm{~mL} \cdot \mathrm{min}^{-1}\right)$ & $3035 \pm 547$ & $1654-4680$ \\
\hline $\mathrm{VT}_{1}\left(\mathrm{~mL} \cdot \mathrm{min}^{-1}\right)$ & $2060 \pm 435$ & $873-3200$ \\
\hline $\mathrm{VO}_{2 \text { peak }}\left(\mathrm{mL} \cdot \mathrm{kg}^{-1} \cdot \mathrm{min}^{-1}\right)$ & $60.0 \pm 4.9$ & $43.1-73.2$ \\
\hline $\mathrm{VT}_{2}\left(\mathrm{~mL} \cdot \mathrm{kg}^{-1} \cdot \mathrm{min}^{-1}\right)$ & $45.3 \pm 4.1$ & $33.9-58.1$ \\
\hline $\mathrm{VT}_{1}\left(\mathrm{~mL} \cdot \mathrm{kg}^{-1} \cdot \mathrm{min}^{-1}\right)$ & $30.7 \pm 4.6$ & $17.7-40.6$ \\
\hline $\mathrm{HR}_{\max }(\mathrm{bpm})$ & $193 \pm 7.0$ & $176-215$ \\
\hline $\mathrm{HR}$ at $\mathrm{VT}_{2}(\mathrm{bpm})$ & $178 \pm 8.0$ & $159-205$ \\
\hline $\mathrm{HR}$ at $\mathrm{VT}_{1}(\mathrm{bpm})$ & $148 \pm 12.0$ & $115-180$ \\
\hline $\operatorname{MAS}\left(\mathrm{km} \cdot \mathrm{h}^{-1}\right)$ & $17.7 \pm 1.6$ & $13-21$ \\
\hline$V T_{2 \text { speed }}\left(\mathrm{km} \cdot \mathrm{h}^{-1}\right)$ & $14.3 \pm 1.1$ & $10-17$ \\
\hline $\mathrm{VT}_{\text {1speed }}\left(\mathrm{km} \cdot \mathrm{h}^{-1}\right)$ & $9.9 \pm 1.1$ & $7-13.5$ \\
\hline
\end{tabular}

Data expressed as mean and standard deviation (mean $\pm \mathrm{SD}$ ). MAS $=$ maximal aerobic speed; $\mathrm{VT}_{\text {2speed }}=$ speed at $\mathrm{VT}_{2} ; \mathrm{VT}_{\text {1speed }}=$ speed at $\mathrm{VT}_{1} ; \mathrm{HR}=$ heart rate.

Table 2. Physical and physiological profile of young elite soccer players according to biological maturation status

\begin{tabular}{lccccc}
\hline Variable & $\begin{array}{c}\text { PREP } \\
(\mathrm{n}=26)\end{array}$ & $\begin{array}{c}\text { PUB } \\
(\mathrm{n}=90)\end{array}$ & $\begin{array}{c}\text { POSP } \\
(\mathrm{n}=85)\end{array}$ & $\eta^{2}$ & ES $_{\mathrm{q}}$ \\
\hline Age (years) & $12.7 \pm 0.8$ & $14.2 \pm 1.4^{\star}$ & $16.8 \pm 1.4^{\star} \#$ & 0.560 & Large \\
Body mass $(\mathrm{kg})$ & $51.3 \pm 8.0$ & $64.9 \pm 9.7^{*}$ & $74.2 \pm 6.4^{\star} \#$ & 0.454 & Large \\
Height $(\mathrm{cm})$ & $161.2 \pm 8.7$ & $171.1 \pm 7.6^{*}$ & $179.0 \pm 6.6^{\star} \#$ & 0.391 & Large \\
Years of training & $4.1 \pm 2.1$ & $5.2 \pm 2.3$ & $6.3 \pm 3.0^{\star} \#$ & 0.077 & Medium \\
$\mathrm{VO}_{2 \text { peak }}\left(\mathrm{mL} \cdot \mathrm{min}^{-1}\right)$ & $2998 \pm 577$ & $3894 \pm 536^{*}$ & $4461 \pm 436^{\star} \#$ & 0.478 & Large \\
$\mathrm{VT}_{2}\left(\mathrm{~mL} \cdot \mathrm{min}^{-1}\right)$ & $2312 \pm 494$ & $2952 \pm 457^{*}$ & $3340 \pm 393^{\star} \#$ & 0.373 & Large
\end{tabular}

Continue... 
... continue

\begin{tabular}{|c|c|c|c|c|c|}
\hline $\mathrm{VT}_{1}\left(\mathrm{~mL} \cdot \mathrm{min}^{-1}\right)$ & $1639 \pm 482$ & $2040 \pm 404^{*}$ & $2210 \pm 366^{\star} \#$ & 0.172 & Large \\
\hline $\mathrm{VO}_{2 \text { peak }}\left(\mathrm{mL} \cdot \mathrm{kg}^{-1} \cdot \mathrm{min}^{-1}\right)$ & $58.5 \pm 5.8$ & $60.2 \pm 5.0$ & $60.3 \pm 4.5$ & 0.015 & Small \\
\hline $\mathrm{VT}_{2}\left(\mathrm{~mL} \cdot \mathrm{kg}^{-1} \cdot \mathrm{min}^{-1}\right)$ & $45.4 \pm 5.5$ & $45.6 \pm 4.4$ & $45.1 \pm 3.4$ & 0.004 & Small \\
\hline $\mathrm{VT}_{1}\left(\mathrm{~mL} \cdot \mathrm{kg}^{-1} \cdot \mathrm{min}^{-1}\right)$ & $31.6 \pm 6.6$ & $31.4 \pm 4.3$ & $29.8 \pm 3.9$ & 0.039 & Small \\
\hline $\mathrm{HR}_{\max }(\mathrm{bpm})$ & $194 \pm 8$ & $193 \pm 7$ & $193 \pm 7$ & 0.002 & Small \\
\hline $\mathrm{HR}$ at VT ${ }_{2}(\mathrm{bpm})$ & $182 \pm 9$ & $179 \pm 8$ & $177 \pm 7^{*}$ & 0.032 & Small \\
\hline $\mathrm{HR}$ at $\mathrm{VT}_{1}(\mathrm{bpm})$ & $154 \pm 13$ & $149 \pm 11$ & $147 \pm 12^{*}$ & 0.045 & Small \\
\hline $\operatorname{MAS}\left(\mathrm{km} \cdot \mathrm{h}^{-1}\right)$ & $15.6 \pm 1.0$ & $17.4 \pm 1.5^{\star}$ & $18.6 \pm 1.1^{\star} \#$ & 0.353 & Large \\
\hline$V T_{2 \text { speed }}\left(\mathrm{km} \cdot \mathrm{h}^{-1}\right)$ & $13.1 \pm 0.9$ & $14.3 \pm 1.1^{*}$ & $14.8 \pm 0.9^{*} \#$ & 0.215 & Large \\
\hline$V T_{1 \text { speed }}\left(\mathrm{km} \cdot \mathrm{h}^{-1}\right)$ & $9.6 \pm 0.8$ & $9.9 \pm 1.1$ & $10.0 \pm 1.1$ & 0.015 & Small \\
\hline
\end{tabular}

Data were expressed as mean and standard deviation (mean $\pm \mathrm{SD})$, where * $=$ significantly different from prepubertal group; \# = significantly different from pubertal group; PREP= prepubertal group; $\mathrm{PUB}=$ pubertal group; $\mathrm{POSP}=$ postpubertal group; $\mathrm{MAS}=$ maximal aerobic speed; $\mathrm{VT}_{\text {2speed }}=$ speed at $\mathrm{VT}_{2} ; \mathrm{VT}_{1 \text { speed }}=$ speed at $\mathrm{VT}_{1} ; \mathrm{HR}=$ heart rate $; \eta^{2}=$ partial eta square; $\mathrm{ES}_{\mathrm{q}}=$ qualitative effect size . Significance $(\mathrm{P}<0.05)$

Table 3. Physical and physiological profile of young elite soccer players according to Tanner stage

\begin{tabular}{|c|c|c|c|c|c|c|c|}
\hline \multirow[b]{2}{*}{ Variable } & \multicolumn{5}{|c|}{ Tanner Stage } & \multirow[b]{2}{*}{$\eta^{2}$} & \multirow[b]{2}{*}{$\mathrm{ES}_{\mathrm{q}}$} \\
\hline & $\begin{array}{c}\text { I } \\
(\mathrm{n}=26)\end{array}$ & $\begin{array}{c}\text { II } \\
(n=13)\end{array}$ & $\begin{array}{c}\text { III } \\
(\mathrm{n}=39)\end{array}$ & $\begin{array}{c}\text { IV } \\
(n=38)\end{array}$ & $\begin{array}{c}V \\
(n=85)\end{array}$ & & \\
\hline Age (years) & $12.7 \pm 0.8$ & $12.9 \pm 1.0$ & $14.2 \pm 1.3^{*} \#$ & $14.7 \pm 1.3^{\star} \#$ & $16.8 \pm 1.4^{\star} \#+\S$ & 0.599 & Small \\
\hline Body mass (kg) & $51.3 \pm 8.0$ & $54.1 \pm 5.0$ & $63.0 \pm 8.5^{\star} \#$ & $70.5 \pm 8.2^{\star} \#+$ & $74.2 \pm 6.4^{\star} \#$ & 0.569 & Small \\
\hline Height (cm) & $161.2 \pm 8.8$ & $162.4 \pm 5.1$ & $170.3 \pm 6.3^{\star} \#$ & $175.0 \pm 7.1^{\star} \# \dagger$ & $179.0 \pm 6.6^{\star} \#+\S$ & 0.478 & Small \\
\hline Years of training & $4.1 \pm 2.1$ & $5.3 \pm 1.9$ & $4.5 \pm 2.2$ & $5.8 \pm 2.5$ & $6.3 \pm 3.0^{*} t$ & 0.097 & Medium \\
\hline $\mathrm{VO}_{2 \text { peak }}\left(\mathrm{mL} \cdot \mathrm{min}^{-1}\right)$ & $2998 \pm 577$ & $3265 \pm 418$ & $3875 \pm 529^{\star} \#$ & $4127 \pm 389^{*} \#$ & $4467 \pm 435^{\star} \#+\S$ & 0.554 & Large \\
\hline $\mathrm{VT}_{2}\left(\mathrm{~mL} \cdot \mathrm{min}^{-1}\right)$ & $2312 \pm 494$ & $2370 \pm 300$ & $2970 \pm 437^{\star} \#$ & $3132 \pm 353^{\star} \#$ & $3344 \pm 391^{\star} \#+$ & 0.467 & Large \\
\hline $\mathrm{VT}_{1}\left(\mathrm{~mL} \cdot \mathrm{min}^{-1}\right)$ & $1639 \pm 483$ & $1572 \pm 249$ & $2050 \pm 346^{\star} \#$ & $2191 \pm 387^{\star} \#$ & $2208 \pm 362^{*} \#$ & 0.270 & Large \\
\hline $\mathrm{VO}_{2 \text { peak }}\left(\mathrm{mL} \cdot \mathrm{kg}^{-1} \cdot \mathrm{min}^{-1}\right)$ & $58.5 \pm 5.7$ & $60.3 \pm 4.3$ & $61.7 \pm 4.2$ & $58.8 \pm 4.7$ & $60.2 \pm 4.9$ & 0.047 & Small \\
\hline $\mathrm{VT}_{2}\left(\mathrm{~mL} \cdot \mathrm{kg}^{-1} \cdot \mathrm{min}^{-1}\right)$ & $45.4 \pm 5.5$ & $43.8 \pm 4.1$ & $47.2 \pm 4.5$ & $44.6 \pm 3.8 \dagger$ & $45.0 \pm 3.4$ & 0.058 & Small \\
\hline $\mathrm{VT}_{1}\left(\mathrm{~mL} \cdot \mathrm{kg}^{-1} \cdot \mathrm{min}^{-1}\right)$ & $31.6 \pm 6.6$ & $29.1 \pm 4.0$ & $32.4 \pm 4.0$ & $31.2 \pm 4.5$ & $29.6 \pm 3.9 t$ & 0.067 & Medium \\
\hline $\mathrm{HR}_{\max }(\mathrm{bpm})$ & $194 \pm 7$ & $199 \pm 8$ & $192 \pm 7$ & $193 \pm 8$ & $193 \pm 7$ & 0.042 & Small \\
\hline $\mathrm{HR}$ at $\mathrm{VT}_{2}(\mathrm{bpm})$ & $182 \pm 9$ & $182 \pm 8$ & $178 \pm 8 \#$ & $178 \pm 8$ & $177 \pm 7$ & 0.046 & Small \\
\hline $\mathrm{HR}$ at $\mathrm{VT}_{1}(\mathrm{bpm})$ & $154 \pm 13$ & $152 \pm 11$ & $148 \pm 10$ & $149 \pm 11$ & $146 \pm 12^{*}$ & 0.051 & Small \\
\hline $\operatorname{MAS}\left(\mathrm{km} \cdot \mathrm{h}^{-1}\right)$ & $15.6 \pm 1.0$ & $16.5 \pm 1.4$ & $17.6 \pm 1.5^{\star}$ & $17.5 \pm 1.5^{\star}$ & $18.6 \pm 1.1^{\star} \#+\S$ & 0.376 & Large \\
\hline $\mathrm{VT}_{\text {2speed }}\left(\mathrm{km} \cdot \mathrm{h}^{-1}\right)$ & $13.0 \pm 0.9$ & $13.6 \pm 1.3$ & $14.5 \pm 0.9^{\star} \#$ & $14.3 \pm 1.2^{*}$ & $14.7 \pm 0.9^{\star} \#$ & 0.249 & Large \\
\hline$V T_{\text {1speed }}\left(\mathrm{km} \cdot \mathrm{h}^{-1}\right)$ & $9.6 \pm 0.8$ & $9.7 \pm 1.1$ & $10.0 \pm 1.0$ & $9.9 \pm 1.1$ & $10.1 \pm 1.1$ & 0.019 & Small \\
\hline
\end{tabular}

Data were expressed as mean and standard deviation (mean $\pm \mathrm{SD}$ ), where * = significantly different from group I; \# = significantly different from group II; $\dagger=$ significantly different from group III; $\S=$ significantly different from group IV; MAS = maximal aerobic speed; $\mathrm{VT}_{2 \text { speed }}=$ speed at $\mathrm{VT}_{2} ; \mathrm{VT}_{1 \text { speed }}=$ speed at $\mathrm{VT}_{1} ; \mathrm{HR}=$ heart rate; $\eta^{2}=$ partial eta square; $\mathrm{ES}_{\mathrm{q}}=$ qualitative effect size. Significance $(\mathrm{P}<0.05)$.

Table 4. Physical and physiological profile of young elite soccer players according to chronological age

\begin{tabular}{|c|c|c|c|c|c|c|}
\hline \multirow[b]{2}{*}{ Variable } & \multicolumn{4}{|c|}{ Chronological Age (years) } & \multirow[b]{2}{*}{$\eta^{2}$} & \multirow[b]{2}{*}{$\mathrm{ES}_{\mathrm{q}}$} \\
\hline & $\begin{array}{c}11-12 \\
(n=26)\end{array}$ & $\begin{array}{c}13-14 \\
(n=68)\end{array}$ & $\begin{array}{c}15-16 \\
(n=58)\end{array}$ & $\begin{array}{c}>17 \\
(n=49)\end{array}$ & & \\
\hline Body mass $(\mathrm{kg})$ & $52.8 \pm 10.1$ & $63.4 \pm 9.7^{*}$ & $71.3 \pm 6.8^{\star} \#$ & $75.0 \pm 6.9^{*} \#$ & 0.438 & Large \\
\hline Height $(\mathrm{cm})$ & $160.6 \pm 8.5$ & $169.9 \pm 7.5^{\star}$ & $177.0 \pm 6.9^{\star} \#$ & $179.7 \pm 6.0^{\star} \#$ & 0.433 & Large \\
\hline Years of training & $4.6 \pm 2.1$ & $4.3 \pm 2.2$ & $6.0 \pm 2.6 \#$ & $7.1 \pm 2.9^{*} \#$ & 0.172 & Large \\
\hline $\mathrm{VO}_{2 \text { peak }}\left(\mathrm{mL} \cdot \mathrm{min}^{-1}\right)$ & $2909 \pm 522$ & $3812 \pm 479^{*}$ & $4346 \pm 404^{\star} \#$ & $4513 \pm 472^{*} \#$ & 0.557 & Large \\
\hline $\mathrm{VT}_{2}\left(\mathrm{~mL} \cdot \mathrm{min}^{-1}\right)$ & $2194 \pm 413$ & $2875 \pm 391^{*}$ & $3307 \pm 328^{\star} \#$ & $3382 \pm 430^{*} \#$ & 0.507 & Large \\
\hline $\mathrm{VT}_{1}\left(\mathrm{~mL} \cdot \mathrm{min}^{-1}\right)$ & $1457 \pm 372$ & $2026 \pm 357^{*}$ & $2261 \pm 310^{\star} \#$ & $2187 \pm 401^{*}$ & 0.335 & Large \\
\hline $\mathrm{VO}_{2 \text { peak }}\left(\mathrm{mL} \cdot \mathrm{kg}^{-1} \cdot \mathrm{min}^{-1}\right)$ & $56.2 \pm 5.1$ & $60.5 \pm 4.6^{*}$ & $61.1 \pm 4.3^{*}$ & $60.2 \pm 4.9^{*}$ & 0.095 & Medium \\
\hline $\mathrm{VT}_{2}\left(\mathrm{~mL} \cdot \mathrm{kg}^{-1} \cdot \mathrm{min}^{-1}\right)$ & $42.3 \pm 4.6$ & $45.7 \pm 3.8^{\star}$ & $46.5 \pm 4.0^{*}$ & $45.0 \pm 3.6^{\star}$ & 0.100 & Medium \\
\hline $\mathrm{VT}_{1}\left(\mathrm{~mL} \cdot \mathrm{kg}^{-1} \cdot \mathrm{min}^{-1}\right)$ & $27.9 \pm 4.9$ & $32.1 \pm 4.5^{\star}$ & $31.8 \pm 3.9^{*}$ & $28.9 \pm 4.1 \# \dagger$ & 0.137 & Medium \\
\hline
\end{tabular}

Continue... 


\begin{tabular}{|c|c|c|c|c|c|c|}
\hline $\mathrm{HR}_{\max }$ (bpm) & $195 \pm 8$ & $193 \pm 7$ & $194 \pm 5$ & $192 \pm 8$ & 0.018 & Small \\
\hline $\mathrm{HR}$ at $\mathrm{VT}_{2}(\mathrm{bpm})$ & $180 \pm 8$ & $179 \pm 8$ & $179 \pm 7$ & $176 \pm 8$ & 0.040 & Small \\
\hline $\mathrm{HR}$ at $\mathrm{VT}_{1}(\mathrm{bpm})$ & $151 \pm 11$ & $150 \pm 12$ & $150 \pm 10$ & $143 \pm 13^{\star} \#+$ & 0.077 & Medium \\
\hline $\operatorname{MAS}\left(\mathrm{km} \cdot \mathrm{h}^{-1}\right)$ & $15.5 \pm 1.2$ & $17.0 \pm 1.2^{*}$ & $18.3 \pm 1.3^{\star} \#$ & $18.9 \pm 0.9^{*} \# \dagger$ & 0.470 & Large \\
\hline $\mathrm{VT}_{\text {2speed }}\left(\mathrm{km} \cdot \mathrm{h}^{-1}\right)$ & $12.9 \pm 1.0$ & $14.1 \pm 0.9^{*}$ & $14.7 \pm 1.1^{\star} \#$ & $15.0 \pm 0.8^{\star} \#$ & 0.339 & Large \\
\hline$V T_{1 \text { speed }}\left(\mathrm{km} \cdot \mathrm{h}^{-1}\right)$ & $9.4 \pm 0.9$ & $9.8 \pm 1.0$ & $10.3 \pm 1.1^{*}$ & $10.0 \pm 1.1$ & 0.067 & Medium \\
\hline
\end{tabular}

Data expressed as mean and standard deviation (mean $\pm \mathrm{SD}$ ), where * ${ }^{*}$ significantly different from 11-12 years old group; \# = significantly different from 13-14 years old group; $\dagger=$ significantly different from 15-16 years old group; $\mathrm{MAS}=$ maximal aerobic speed; $\mathrm{VT}_{2 \text { speed }}=$ speed at $\mathrm{VT}_{2} ; \mathrm{VT}_{\text {1speed }}=$ speed at $\mathrm{VT}_{1} ; \mathrm{HR}=$ heart rate; $\eta^{2}=$ partial eta square; $\mathrm{ES}_{\mathrm{q}}=$ qualitative effect size. Significance $(\mathrm{P}<0.05)$.

Table 5. Physical and physiological profile of young elite soccer players in according to playing position

\begin{tabular}{|c|c|c|c|c|c|c|c|c|}
\hline \multirow[b]{2}{*}{ Variable } & \multicolumn{6}{|c|}{ Playing Position } & \multirow[b]{2}{*}{$\eta^{2}$} & \multirow[b]{2}{*}{$\mathrm{ES}_{\mathrm{q}}$} \\
\hline & $\begin{array}{l}\mathrm{GK} \\
(\mathrm{n}=14)\end{array}$ & $\begin{array}{l}F B \\
(n=35)\end{array}$ & $\begin{array}{l}\mathrm{CB} \\
(\mathrm{n}=33)\end{array}$ & $\begin{array}{l}\text { DM } \\
(\mathrm{n}=37)\end{array}$ & $\begin{array}{l}O M \\
(n=40)\end{array}$ & $\begin{array}{l}\text { AT } \\
(\mathrm{n}=36)\end{array}$ & & \\
\hline Age (years) & $14.4 \pm 2.0$ & $14.8 \pm 2.1$ & $15.2 \pm 1.9$ & $15.6 \pm 2.0$ & $15.1 \pm 1.9$ & $15.4 \pm 2.3$ & 0.025 & Small \\
\hline Body mass (kg) & $69.8 \pm 12.8$ & $61.9 \pm 10.2$ & $75.0 \pm 8.1 \#$ & $70.9 \pm 9.2 \#$ & $62.0 \pm 9.0+\S$ & $65.7 \pm 12.6 \dagger$ & 0.193 & Large \\
\hline Height (cm) & $177.8 \pm 12.7$ & $168.9 \pm 8.4^{\star}$ & $180.5 \pm 6.2 \#$ & $175.0 \pm 6.6 \#$ & $168.9 \pm 6.6^{*}+\S$ & $171.9 \pm 11.7 \dagger$ & 0.211 & Large \\
\hline Years of training & $5.7 \pm 2.2$ & $5.5 \pm 2.7$ & $5.2 \pm 2.6$ & $5.8 \pm 2.7$ & $6.3 \pm 3.1$ & $5.0 \pm 2.3$ & 0.029 & Small \\
\hline $\mathrm{VO}_{2 \text { peak }}\left(\mathrm{mL} \cdot \mathrm{min}^{-1}\right)$ & $4028 \pm 807$ & $3735 \pm 678$ & $4381 \pm 457 \#$ & $4272 \pm 634 \#$ & $3791 \pm 555+\S$ & $3975 \pm 846$ & 0.124 & Medium \\
\hline $\mathrm{VT}_{2}\left(\mathrm{~mL} \cdot \mathrm{min}^{-1}\right)$ & $2930 \pm 622$ & $2832 \pm 550$ & $3318 \pm 371 \#$ & $3277 \pm 535 \#$ & $2842 \pm 410+\S$ & $2983 \pm 652$ & 0.134 & Medium \\
\hline $\mathrm{VT}_{1}\left(\mathrm{~mL} \cdot \mathrm{min}^{-1}\right)$ & $1980 \pm 428$ & $1836 \pm 436$ & $2280 \pm 291 \#$ & $2222 \pm 427 \#$ & $1948 \pm 363 t$ & $2019 \pm 504$ & 0.134 & Medium \\
\hline $\mathrm{VO}_{2 \text { peak }}\left(\mathrm{mL} \cdot \mathrm{kg}^{-1} \cdot \mathrm{min}^{-1}\right)$ & $57.2 \pm 5.7$ & $60.3 \pm 4.9$ & $58.8 \pm 5.6$ & $60.3 \pm 4.4$ & $61.3 \pm 4.5$ & $60.4 \pm 4.5$ & 0.051 & Small \\
\hline $\mathrm{VT}_{2}\left(\mathrm{~mL} \cdot \mathrm{kg}^{-1} \cdot \mathrm{min}^{-1}\right)$ & $41.9 \pm 3.3$ & $45.7 \pm 4.8^{\star}$ & $44.4 \pm 4.0$ & $46.1 \pm 3.8^{*}$ & $46.2 \pm 4.0^{\star}$ & $45.3 \pm 3.8$ & 0.077 & Medium \\
\hline $\mathrm{VT}_{1}\left(\mathrm{~mL} \cdot \mathrm{kg}^{-1} \cdot \mathrm{min}^{-1}\right)$ & $28.3 \pm 3.1$ & $29.5 \pm 5.4$ & $30.4 \pm 3.9$ & $31.3 \pm 3.9$ & $31.7 \pm 5.0$ & $30.6 \pm 4.6$ & 0.044 & Small \\
\hline $\mathrm{HR}_{\text {max }}(\mathrm{bpm})$ & $192 \pm 6$ & $195 \pm 8$ & $193 \pm 6$ & $195 \pm 7$ & $194 \pm 8$ & $192 \pm 7$ & 0.025 & Small \\
\hline $\mathrm{HR}$ at $\mathrm{VT}_{2}(\mathrm{bpm})$ & $176 \pm 7$ & $180 \pm 8$ & $178 \pm 7$ & $179 \pm 8$ & $179 \pm 9$ & $178 \pm 8$ & 0.021 & Small \\
\hline $\mathrm{HR}$ at $\mathrm{VT}_{1}(\mathrm{bpm})$ & $145 \pm 9$ & $148 \pm 12$ & $146 \pm 11$ & $150 \pm 11$ & $149 \pm 13$ & $150 \pm 13$ & 0.022 & Small \\
\hline $\operatorname{MAS}\left(\mathrm{km} \cdot \mathrm{h}^{-1}\right)$ & $16.6 \pm 2$ & $17.7 \pm 1.6$ & $17.8 \pm 1.4$ & $18.2 \pm 1.3^{*}$ & $17.7 \pm 1.7$ & $17.5 \pm 1.5$ & 0.053 & Small \\
\hline$V T_{\text {2speed }}\left(\mathrm{km} \cdot \mathrm{h}^{-1}\right)$ & $13.0 \pm 1.3$ & $14.6 \pm 1.0^{*}$ & $14.4 \pm 1.1^{*}$ & $14.6 \pm 1.0^{*}$ & $14.4 \pm 1.1^{*}$ & $14.2 \pm 1.0^{*}$ & 0.119 & Medium \\
\hline$V T_{\text {Ispeed }}\left(\mathrm{km} \cdot \mathrm{h}^{-1}\right)$ & $9.5 \pm 0.9$ & $10.2 \pm 1.2$ & $10.0 \pm 1.2$ & $9.9 \pm 1.1$ & $10.1 \pm 0.9$ & $9.6 \pm 0.9$ & 0.045 & Small \\
\hline
\end{tabular}

Data expressed as mean and standard deviation (mean $\pm \mathrm{SD}$ ), where * = significantly different from goalkeeper group; \# = significantly different from full-back group; $\dagger=$ significantly different central-back group; $\S=$ significantly different from defensive midfielder group; $\mathrm{GK}=$ goalkeeper; $\mathrm{FB}=$ full-back; $\mathrm{CB}=$ central-back; $\mathrm{DM}=$ defensive midfielder; $\mathrm{OM}=$ offensive midfielder; $\mathrm{AT}=$ attacker; $\mathrm{MAS}=$ maximal aerobic speed; $\mathrm{VT}_{2 \text { speed }}=$ speed at $\mathrm{VT}_{2} ; \mathrm{VT}_{1 \text { speed }}=$ speed at $\mathrm{VT}_{1} ; \mathrm{HR}=$ heart rate; $\eta^{2}=$ partial eta square; $\mathrm{ES}_{q}=$ qualitative effect size; Significance $(\mathrm{P}<0.05)$.

\section{DISCUSSION}

This study presented a profile of physical and physiological features for highlevel young soccer players and identified the effects of biological maturation, chronological age, RAE and playing position on aerobic fitness parameters $\left(\mathrm{VO}_{2 \text { peak }}, \mathrm{VT}_{2}, \mathrm{VT}_{1}, \mathrm{MAS}, \mathrm{VT}_{2 \text { speed }}\right.$ and $\left.\mathrm{VT}_{\text {1speed }}\right)$. These variables have been described as important intervenient factors for the talent development and/ or talent identification process as well for soccer performance.

To our knowledge, this was the first study to report the effects of biological maturation, chronological age, RAE and playing position on aerobic fitness profile directly assessed by ergospirometry with a large sample and age range of high-level young soccer player's simultaneously. The main findings from the present study were as follows: 1) biological maturation status showed no significant effects on $\mathrm{VO}_{2 \text { peak }}, \mathrm{VT}_{2}$, and $\mathrm{VT}_{1}$ relative to 
body size values $\left(\mathrm{mL} \cdot \mathrm{kg}^{-1} \cdot \mathrm{min}^{-1}\right)$ in young soccer players; 2$)$ chronological age showed a medium positive effect on $\mathrm{VO}_{2 \text { peak }}, \mathrm{VT}_{2}$, and $\mathrm{VT}_{1}\left(\mathrm{~mL}^{\mathrm{kg}} \mathrm{k}^{-1}\right.$ $\min ^{-1}$ ) relative values; 3) RAE was confirmed since there was an overrepresentation (76.2\%) of players born in the first semester of the year; 4) playing position showed no significant differences among groups for $\mathrm{VO}_{2 \text { peak }}$ and $V_{1}\left(\mathrm{~mL}_{1} \mathrm{~kg}^{-1} \cdot \mathrm{min}^{-1}\right)$ relative values, but showed medium positive effect on $\left.\mathrm{VT}_{2}\left(\mathrm{~mL} \mathrm{~kg}^{-1} \cdot \mathrm{min}^{-1}\right) ; 5\right)$ biological maturation and chronological age showed a large positive effect on MAS and $\mathrm{VT}_{2 \text { speed, }}$, while playing position showed a medium positive effect only $\mathrm{VT}_{\text {2speed. }}$

Programs developed for detection or development of players with potential talent can be biased by the intervenient effects of biological maturation, relative age, and playing position. As a result, potentially talented players can systematically drop out the sport at an early age, and not reach higher levels of competition ${ }^{12,26}$. In the present study, biological maturation status did not show a significant positive effect on aerobic fitness parameters $\left(\mathrm{VO}_{2 \text { peak }}, \mathrm{VT}_{2}, \mathrm{VT}_{1}\right.$ express in $\left.\mathrm{mL} \cdot \mathrm{kg}^{-1} \cdot \mathrm{min}^{-1}\right)$ in young soccer players. Some studies also did not find positive effects of biological maturation on aerobic fitness parameters of young soccer players ${ }^{6,16-18,21,27}$. However, other studies demonstrated positive effects of biological maturation status on aerobic fitness parameters of young soccer player ${ }^{9-11}$. The contrasting findings regarding effects of biological maturation on aerobic fitness parameters may be attributed to differences in the sample size experimental design (i.e. different methods used to assess aerobic fitness), scaling variable to normalize aerobic fitness (i.e. absolute values, relative to body mass, relative to fat-free mass), and methods for estimating biological maturation (i.e. Tanner stages, peak high velocity) ${ }^{6,10,18,21}$. The effect of biological maturation status on aerobic fitness parameters seems inconclusive for young soccer players. It is important to highlight that body mass $(\mathrm{kg})$ is a crucial variable that has a large impact on athletic performance. The normalization of aerobic fitness parameters for body mass has been broadly used over time due to the high correlation with $\mathrm{VO}_{2 \text { peak }}$. For this reason, absolute $\mathrm{VO}_{2 \text { peak }}, \mathrm{VT}_{2}$, and $\mathrm{VT}_{1}(\mathrm{~mL}$.min-1) values are not considered appropriated to compare young soccer players with diverse biological maturation status, chronological age and/or body size $\mathrm{e}^{6,11,21}$.

Chronological age showed a medium positive effect on aerobic fitness parameters. Positive effects were observed for $\mathrm{VO}_{2 \text { peak, }} \mathrm{VT}_{2}$, and $\mathrm{VT}_{1}(\mathrm{~mL}$. $\left.\mathrm{kg}^{-1} \cdot \mathrm{min}^{-1}\right)$ manly due significant differences among the 13-14, 15-16 and $>17$ years-old groups compared to the $11-12$ years-old group. This result corroborate with previous finding ${ }^{26,27}$. These studies showed that aerobic fitness parameters increase progressively from 8 to 18 years of age. However, Cunha et al. ${ }^{6}$ using multiple linear regression analysis showed that chronological age did not show positive effect on $\mathrm{VO}_{2 \text { peak }}, \mathrm{VT}_{2}$ and $\mathrm{VT}_{1}$ $\left(\mathrm{mL} \cdot \mathrm{kg}^{-1} \cdot \mathrm{min}^{-1}\right)$ in young soccer player.

In the present study, an overrepresentation of players born during the first semester of the year was observed, confirming the phenomenon known as RAE. Birth-date distribution by month quartile was $49.8 \%, 26.4 \%$, 
$15.9 \%$ and $8 \%$ for $\mathrm{BQ}_{4}, \mathrm{BQ}_{2}, \mathrm{BQ}_{2}$, and $\mathrm{BQ}_{4}$, respectively. Similar findings were shown in early studies ${ }^{13,16,28}$. It has been well accepted that players born in the first semester of the year are usually taller, heavier and have greater aerobic power, muscular strength, speed, perception of competence, and self-efficacy compared with their relatively younger peers (born in the last semester of the year ${ }^{14,16,23,28}$. RAE has been associated with success in youth soccer players. There were positive correlations among RAE and rank, points scored, and goals against in U-17 Germany soccer teams ${ }^{29}$. In addition, Carling et al. ${ }^{23}$ showed that out of a total of $75,49,26$, and 10 players born in the $\mathrm{BQ}_{4}, \mathrm{BQ}_{2}, \mathrm{BQ}_{3}$, and $\mathrm{BQ}_{4}$ periods, $45.6 \%, 38.8 \%$, $43.3 \%$ and $70 \%$ achieved professional status, respectively. Interestingly, a meta-analytical review identified consistent prevalence of RAE in some sports (ice-rocky, volleyball, basketball, football, and soccer), but with small effect size ${ }^{14}$.

Playing position can also to exert effect on aerobic fitness parameters in young soccer players ${ }^{3,4,19,20}$. A large association has been observed between aerobic fitness and total distance covered during a match in youth soccer players ${ }^{3,7}$. More specifically, relationship between match running performance and aerobic fitness parameters are more position-dependent ${ }^{3,4}$. In general, midfielders and attackers show higher values of aerobic fitness and covered more distance in highest intensity zone compared to defensive players ${ }^{3,4}$. In the present study, playing position showed no significant effects on $\mathrm{VO}_{2 \text { peak }}\left(\mathrm{mL} \cdot \mathrm{kg}^{-1} \cdot \mathrm{min}^{-1}\right)$ among GK, FB, CB, DM, OM and AT groups. In additional, other studies showed that aerobic endurance $(\mathrm{m})$ was different among playing positions. Indeed goalkeepers showed lower values compared to defenders, midfielders and attackers ${ }^{19,20}$. However, Coelho \& Silva et al. ${ }^{22}$ also did not find effects of playing position on aerobic endurance $(m)$ in young Portuguese soccer players from local and regional levels. The discrepancies among these results might be partly explained by the use of different methods to determine aerobic fitness parameters (direct vs. indirect tests), by the complexity nature of physical demands during a game, and also by the playing position-specific running pattern (such as the link between defense and attack, technical actions, change positions, covered distance in low, moderate or high-intensity, acceleration for create space and counter-attack) ${ }^{3,4,19,20,22}$.

Few studies have assessed the effects of playing position on ventilatory thresholds in young soccer player. In the present study, GK showed $\mathrm{VT}_{2}\left(\mathrm{~mL}^{\mathrm{kg}}{ }^{-1} \mathrm{~min}^{-1}\right)$ values significantly lower compared to $\mathrm{FB}, \mathrm{DM}$ and $\mathrm{OF}$ groups. However, $\mathrm{VT}_{1}$ was similar among groups. It can be hypothesized that the observed results can be partially attributed to differences in physical and physiological demands of game among different playing positions (e.g. total distance covered during the match in low, moderate and high-intensity), as well as the position-specific training for goalkeepers, defenders, midfielders and attackers.

There is a gap in the literature about the effects of biological maturation, chronological age and playing position on MAS, $\mathrm{VT}_{2 \text { speed }}$ and $\mathrm{VT}_{\text {1speed }}$. 
MAS has been considered the most powerful determinant of physical game performance in young soccer players ${ }^{3}$. In the present study, biological maturation and chronological age showed a large positive effect while playing position no showed effect on MAS. With respect to MAS, similar results have been reported ${ }^{3,4,30}$. In general, older and more mature players (15 to 18 years old) are faster according to the estimated MAS values (15 to $18 \mathrm{~km} \cdot \mathrm{hr}^{-1}$ ) in comparison to young and less mature players (12 to 15 years old) counterparts (13 to $\left.15 \mathrm{~km}^{-1} \mathrm{hr}^{-1}\right)^{3,4,30}$. In addition, playing position showed no significant effect on $\mathrm{MAS}^{3,4,30}$. It is noteworthy that our results directly assessed of MAS are approximately $1 \mathrm{~km} \cdot \mathrm{hr}^{-1}$ higher than those indirectly values previously reported in function of biological maturation and chronological age.

Based in the common belief that older players are more mature and exhibit better performance at the selection time, coaches tend to prefer those players that achieve success in highly competitive youth sports ${ }^{29}$. However, from the long-term development talent perspective (i.e. development program from youth athlete to elite professional athlete) this selection model might be a concern. A recent longitudinal study ${ }^{12}$ showed that late maturing soccer players are more likely to achieve success in the top level game (60.1\%), compared with their normal maturing (38.1\%) and early maturing counterparts (11.8\%). Additionally, athletes born in the BQ have reached the professional level in a greater proportion than athletes born in $\mathrm{BQ}_{4}, \mathrm{BQ}_{2}$ and $\mathrm{BQ}_{3}{ }^{23}$.

From a practical point of view, coaches, sport scientists and sport administrators should take into account the biological maturation status, $\mathrm{RAE}$ and playing position effects on aerobic fitness profile during talent development and/or talent identification programs, in order to avoid dropout of the potential successful young players. Since MAS and $\mathrm{VT}_{2 \text { speed }}$ have been associated with match running performance in soccer, these variables could too be used to help talent identification/development process and to design specific training programs focused on position-dependent highintensity traits. In addition, caution must be taken when interpreting the effects of biological maturation, chronological age and playing position on aerobic fitness parameters in young soccer players on talent identification/ development perspective. We cannot to assume that important factors of success in professional players automatically can be extrapolated to young soccer players.

\section{CONCLUSION}

In the present study, biological maturation showed no positive effects on aerobic fitness parameters except for MAS and $\mathrm{VT}_{\text {2speed }}$. On the other hand, chronological age showed positive effects on aerobic fitness parameters and an overrepresentation of players born during the first semester of year was found. Finally, playing position showed a positive effect on $\mathrm{VT}_{2}$ and $\mathrm{VT}_{\text {2speed }}$. 


\section{Acknowledgements}

The authors GSC, GTL, MAV and ARO were supported by a CNPq scholarship, and ALL and JMG by a CAPES scholarship.

\section{REFERENCES}

1. Stolen T, Chamari K, Castagna C, Wisloff U. Physiology of soccer: an update. Sports Med 2005;35(6):501-36.

2. Helgerud J, Engen LC, Wisloff U, Hoff J. Aerobic endurance training improves soccer performance. Med Sci Sports Exerc 2001;33(11):1925-31.

3. Buchheit M, Mendez-Villanueva A, Simpson BM, Bourdon PC. Match running performance and fitness in youth soccer. Int J Sports Med 2010;31(11):818-25.

4. Mendez-Villanueva A, Buchheit M, Simpson B, Bourdon PC. Match play intensity distribution in youth soccer. Int J Sports Med 2013;34(2):101-10.

5. Abt G, Lovell R. The use of individualized speed and intensity thresholds for determining the distance run at high-intensity in professional soccer. J Sports Sci 2009;27(9):893-8.

6. Cunha G, Lorenzi T, Sapata K, Lopes AL, Gaya AC, Oliveira A. Effect of biological maturation on maximal oxygen uptake and ventilatory thresholds in soccer players: An allometric approach. J Sports Sci 2011;29(10):1029-39.

7. Castagna C, Impellizzeri F, Cecchini E, Rampinini E, Alvarez JC. Effects of intermittent-endurance fitness on match performance in young male soccer players. J Strength Cond Res 2009;23(7):1954-9.

8. Armstrong N, Welsman JR. Peak oxygen uptake in relation to growth and maturation in 11- to 17-year-old humans. Eur J Appl Physiol 2001;85(6):546-51.

9. Malina RM, Eisenmann JC, Cumming SP, Ribeiro B, Aroso J. Maturity-associated variation in the growth and functional capacities of youth football (soccer) players 13-15 years. Eur J Appl Physiol 2004;91(5-6):555-62.

10. Valente-Dos-Santos J, Coelho ESMJ, Tavares OM, Brito J, Seabra A, Rebelo A, et al. Allometric modelling of peak oxygen uptake in male soccer players of 8-18 years of age. Ann Hum Biol 2015;42(2):125-33.

11. Cunha GS, Célia FG, Ribeiro JL, Oliveira AR. Effects of the biological maturation on maximal oxygen uptake and ventilatory breakpoint of Brazilian soccer players Gazz Med Ital - Arch Sci Med 2008;167(2):43-9.

12. Ostojic SM, Castagna C, Calleja-Gonzalez J, Jukic I, Idrizovic K, Stojanovic M. The biological age of 14-year-old boys and success in adult soccer: do early maturers predominate in the top-level game? Res Sports Med 2014;22(4):398-407.

13. Deprez D, Coutts AJ, Fransen J, Deconinck F, Lenoir M, Vaeyens R, et al. Relative age, biological maturation and anaerobic characteristics in elite youth soccer players. Int J Sports Med 2013;34(10):897-903.

14. Cobley S, Baker J, Wattie N, McKenna J. Annual age-grouping and athlete development: a meta-analytical review of relative age effects in sport. Sports Med 2009;39(3):235-56.

15. Andronikos G, Elumaro AI, Westbury T, Martindale RJ. Relative age effect: implications for effective practice. J Sports Sci 2016;34(12):1124-31.

16. Buchheit M, Mendez-Villanueva A, Mayer N, Jullien H, Marles A, Bosquet L, et al. Locomotor performance in highly-trained young soccer players: does body size always matter? Int J Sports Med 2014;35(6):494-504.

17. Buchheit M, Mendez-Villanueva A. Reliability and stability of anthropometric and performance measures in highly-trained young soccer players: effect of age and maturation. J Sports Sci 2013;31(12):1332-43.

18. Wrigley RD, Drust B, Stratton G, Atkinson G, Gregson W. Long-term soccerspecific training enhances the rate of physical development of academy soccer players independent of maturation status. Int J Sports Med 2014;35(13):1090-4. 
19. Lago-Penas C, Casais L, Dellal A, Rey E, Dominguez E. Anthropometric and physiological characteristics of young soccer players according to their playing positions: relevance for competition success. J Strength Cond Res 2011;25(12):3358-67.

20. Deprez D, Fransen J, Boone J, Lenoir M, Philippaerts R, Vaeyens R. Characteristics of high-level youth soccer players: variation by playing position. J Sports Sci 2015;33(3):243-54.

21. Cunha GS, Vaz MA, Geremia JM, Leites GT, Baptista RR, Lopes AL, et al. Maturity Status Does Not Exert Effects on Aerobic Fitness in Soccer Players After Appropriate Normalization for Body Size. Pediatr Exerc Sci 2016;28(3):456-65.

22. Coelho ESMJ, Figueiredo AJ, Simoes F, Seabra A, Natal A, Vaeyens R, et al. Discrimination of $\mathrm{u}-14$ soccer players by level and position. Int J Sports Med 2010;31(11):790-6.

23. Carling C, le Gall F, Reilly T, Williams AM. Do anthropometric and fitness characteristics vary according to birth date distribution in elite youth academy soccer players? Scand J Med Sci Sports 2009;19(1):3-9.

24. Tanner JM. Growth at Adolescence. 2nd edn. Oxford: Blackwell Scientific Publications, 1962.

25. Cohen J. Statistical Power Analysis for the Behavioral Sciences. Hillsdale: Lawrence Erlbaum; 1988.

26. Figueiredo AJ, Goncalves CE, Coelho ESMJ, Malina RM. Youth soccer players, 11-14 years: maturity, size, function, skill and goal orientation. Ann Hum Biol 2009;36(1):60-73.

27. Figueiredo AJ, Coelho ESMJ, Malina RM. Predictors of functional capacity and skill in youth soccer players. Scand J Med Sci Sports 2011;21(3):446-54.

28. Gil SM, Badiola A, Bidaurrazaga-Letona I, Zabala-Lili J, Gravina L, SantosConcejero J, et al. Relationship between the relative age effect and anthropometry, maturity and performance in young soccer players. J Sports Sci 2014;32(5):479-86.

29. Augste C, Lames M. The relative age effect and success in German elite U-17 soccer teams. J Sports Sci 2011;29(9):983-7.

30. Buchheit M, Mendez-Villanueva A. Effects of age, maturity and body dimensions on match running performance in highly trained under-15 soccer players. J Sports Sci 2014;32(13):1271-8.

\section{CORRESPONDING AUTHOR}

Giovani dos Santos Cunha Universidade Federal do Rio Grande do Sul.

Escola de Educação Física,

Fisioterapia e Dança.

Rua Felizardo, $n^{0} 750$ - Jardim

Botânico - Porto Alegre, RS. Brasil

CEP: $90690-200$

Email: giovani.cunha@ufrgs.br 